Pacific Journal of Mathematic 


\section{NONCOMPLETENESS OF THE WEIL-PETERSSON METRIC FOR TEICHMÜLLER SPACE}

\section{SCOTT WOLPERT}

Let $T_{g}$ be the Teichmuiller space of a compact Riemann surface $R$ of genus $g$ with $g \geqq 2$. In the present paper it is shown thatthe Weil-Petersson length of a large class of rays is finite, deduced that the metric is not complete and indicated how the proof can be extended to the Teichmüller space of an arbitrary finitely generated Fuchsian group of the first kind. The proof is carried out by estimating the Weil-Petersson length of Teichmuiller geodesic rays in directions corresponding to a certain class of quadratic differentials.

Metrics dealing with various properties of $T_{g}$ have been defined. Among them are the Teichmüller, Kobayashi, Carathéodory, Bergman, and Weil-Petersson metrics. Royden has shown that the Teichmüller and Kobayashi metrics coincide, [8]. The Teichmüller-Kobayashi and Carathéodory metrics are known to be complete. Furthermore the Weil-Petersson metric is Hermitian, Kähler and has negative holomorphic sectional curvature, [3].

A trajectory of a quadratic differential $\varphi d z^{2}$ of $R$ is a curve along which $\varphi d z^{2}>0$. Zeros (or poles) of $\varphi d z^{2}$ are referred to as critical points and a trajectory meeting such points as a critical trajectory. By a Jenkins-Strebel differential we mean a quadratic differential $\varphi d z^{2}$ such that the complement on $R$ of the critical trajectories of $\varphi d z^{2}$ is a finite union of conformal annuli $A_{j}, 1<\left|z_{j}\right|<$ $\exp \left(\left|c_{j}\right|^{-1 / 2} \log r_{j}\right)$ with $\varphi d z^{2}=c_{j}\left(d z_{j} \mid z_{j}\right)^{2}$ on $A_{j}$ where $c_{j}<0, j=1, \cdots, n$. The existence of such differentials on finite Riemann surfaces is a consequence of the solution by J. Jenkins of a class of free homotopy a conmodule problems, [7]. A. Douady and J. Hubbard recently confirmed jecture of $\mathrm{K}$. Strebel that such differentials represent a dense subset of the space of all analytic quadratic differentials, [6].

It has been communicated to the author that Mr. T. C. Chu of Columbia University has found a similar result. The author would like to take this opportunity to thank professors Clifford Earle and Halsey Royden for their patience and assistance with this investigation.

1. Description of the curve and its tangent vectors. A path leading to the boundary of the Teichmüller space is given by surfaces $R_{t}$ that are determined by the Beltrami differential $((t-1) /(t+1))$ $\left.\overline{\left(\varphi d z^{2}\right.} /\left|\varphi d z^{2}\right|\right)$, where $t \geqq 1$ and $-\varphi d z^{2}$ is a Jenkins-Strebel differential. 
In a neighborhood of a point $z_{0}$ which is not a zero of $\varphi d z^{2}$ the local coordinates on $R_{t}$ are given by

$$
z_{t}=\left(\int_{z 0}^{z} \sqrt{\varphi d z^{2}}+\frac{t-1}{t+1} \int_{z 0}^{z} \sqrt{\overline{\varphi d z^{2}}}\right)\left(1-\frac{t-1}{t+1}\right)^{-1} .
$$

If $S_{j}$ is a vertical strip in the $\zeta_{j}$ plane s.t. $z_{j}=\exp \left(\left|c_{j}\right|^{-1 / 2} \zeta_{j}\right)$ is a covering of the annulus $A_{j}$ then the formula becomes

$$
\zeta_{j, t}=\left(\zeta_{j}+\frac{t-1}{t+1} \bar{\zeta}_{j}\right)\left(1-\frac{t-1}{t+1}\right)^{-1}, \quad 0<\operatorname{Re} \zeta_{j}<\log r_{j}
$$

or equivalently $\zeta_{j, t}=t \xi_{j}+i \eta_{j}$ where $\zeta_{j}=\xi_{j}+i \eta_{j}$ and $\left(d \zeta_{j, t}\right)^{2}$ is the quadratic differential on $S_{j, t}$ associated with this mapping in the sense of Teichmüller's theorem. By (1) we view the strip $S_{j, t}, 0<\operatorname{Re} \zeta_{j, t}<$ $t \log r_{j}$ in the $\zeta_{j, t}$ plane as the quasiconformal image of the strip $0<\operatorname{Re} \zeta_{j}<\log r_{j}$ in the $\zeta_{j}$ plane. The $S_{j, t}$ cover annuli $A_{j, t}$ by the maps $z_{j, t}=\exp \left(\left|c_{j}\right|^{-1 / 2} \zeta_{j, t}\right)$ and due to the nature of the definition of the new coordinates it is clear that the annuli $A_{j, t}$ are identified to form $R_{t}$ in the same manner in which the $A_{j}$ are identified to form $R$.

It will be necessary to know the tangents to the curve expressed as tangent vectors based at the points $R_{t}$ of Teichmüller space, [2], let $f_{t}$ be the map from the $\zeta$ plane to the $\zeta_{t}$ plane defined by $\zeta_{t}=$ $t \xi+i \eta$. We are interested in the map $f^{\rho}$ from the $\zeta_{t}$ plane to the $\zeta_{\tau}$ plane satisfying $f_{\tau}=f^{\rho} \circ f_{t}$. Clearly $f^{\rho}\left(\zeta_{t}\right)$ is defined by $f^{\rho}\left(\zeta_{t}\right)=$ $(\tau / t) \xi_{t}+i \eta_{t}$ where $\zeta_{t}=\xi_{t}+i \eta_{t}$ or equivalently

$$
f^{\rho}\left(\zeta_{t}\right)=\left(\zeta_{t}+\frac{\tau-t_{\bar{\zeta}_{t}}}{\tau+t}\right)\left(1-\frac{\tau-t}{\tau+t}\right)^{-1} .
$$

It is clear that this is the Teichmüller map associated with Beltrami differential $((\tau-t) /(\tau+t)) \overline{\left(d \zeta_{t}\right)^{2}} /\left|\left(d \zeta_{t}\right)^{2}\right|$. Taking the $\tau$ derivative of $(\tau-t) \backslash(\tau+t)$ and setting $\tau=t$ yields the quantity $1 / 2 t$. Hence the Beltrami differential $(1 / 2 t) \overline{\left(d \zeta_{t}\right)^{2}} /\left|\left(d \zeta_{t}\right)^{2}\right|$ is the tangent to the curve based at the point $R_{t}$.

2. The finite length estimate. For a compact Riemann surface $S$ of genus $g, g \geqq 2$, one can identify the cotangent space at the point $S$ of Teichmüller space with the regular quadratic differentials of $S$, and the tangent space at $S$ with the Beltrami differentials modulo those which are infinitesimally trivial, [2]. The Weil-Petersson cometric is induced by the norm $\|\varphi\|=\left(\int_{s}|\varphi|^{2} \lambda_{s}^{-2}\right)^{1 / 2}$ on the space of regular quadratic differentials $\varphi$ of $S$, where $\lambda_{s}$ is the Poincaré metric for $S$. The Weil-Petersson metric is induced by the norm $\|\mu\|=\sup _{\varphi}|[\mu, \varphi]| /\|\varphi\|$, where $\mu$ is a Beltrami differential, $\varphi$ 
ranges over the regular quadratic differentials of $S$ and $[\mu, \varphi]=$ $\int_{s} \mu \varphi,[10]$. For all $t, t \geqq 1$, it is clear that the union of the regions $0<\operatorname{Re} \zeta_{t}<t \log r_{j}, 0<\operatorname{Im} \zeta_{t}<\left|c_{j}\right|^{1 / 2} 2 \pi$ can be considered as a domain in $R_{t}$ and that its complement on the surface is a set of smooth curves. Hence area integrals for $R_{t}$ can be computed on this union of regions. Since the annulus obtained by taking the quotient of the strip $0<\operatorname{Re} \zeta_{t}<t \log r_{j}$ by the group $\left\{\zeta_{t} \rightarrow \zeta_{t}+n\left|c_{j}\right|^{1 / 2} 2 \pi i, n \in Z\right\}$ is contained in $R_{t}$, the Poincare metric for the annulus bounds the Poincaré metric of $R_{t}$ restricted to the annulus. If $\|\varphi\|_{t}$ is the WeilPetersson norm of a quadratic differential of $R_{t}$, we have

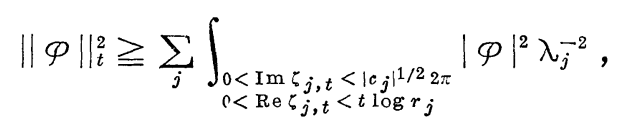

where $\lambda_{j}$ is the Poincaré metric for the $S_{j, t}$, and consequently

$$
\left\|\frac{1}{2 t} \frac{\overline{\left(d \zeta_{t}\right)^{2}}}{\left|\left(d \zeta_{t}\right)^{2}\right|}\right\|_{t}=\sup _{\varphi} \frac{\left|\left[\frac{1}{2 t} \frac{\overline{\left(d \zeta_{t}\right)^{2}}}{\left|\left(d \zeta_{t}\right)^{2}\right|}, \varphi\right]\right|}{\|\varphi\|_{t}} \leqq \sup _{\varphi} \frac{\left|\left[\frac{1}{2 t} \frac{\overline{\left(d \zeta_{t}\right)^{2}}}{\left|\left(d \zeta_{t}\right)^{2}\right|}, \varphi\right]\right|}{\left(\sum_{j} \int|\varphi|^{2} \lambda_{j}^{-2}\right)^{1 / 2}}
$$

Noting that

$$
\lambda_{j}=\frac{\pi}{t \log r_{j}} \csc \frac{\pi}{t \log r_{j}} \operatorname{Re} \zeta_{j, t}\left|d \zeta_{j, t}\right|
$$

and that the metric does not depend on $\operatorname{Im} \zeta_{j, t}$, we see that the extremal value of the last quotient is estimated by $\varphi=\left(d \zeta_{t}\right)^{2}$. Since the $\lambda_{j}$ are known, the quantity

$$
\sum_{j} \int\left|\left(d \zeta_{j, t}\right)^{2}\right|^{2} \lambda_{j}^{-2}
$$

can be computed directly, or the dependence on $t$ can be determined by considering the invariance properties of the $\lambda_{j}$ in either case

$$
\sum_{j} \int\left|\left(d \zeta_{j, t}\right)^{2}\right|^{2} \lambda_{j}^{-2}=C t^{3}, \quad C>0 .
$$

Furthermore we have

$$
\begin{aligned}
{\left[\frac{1}{2 t} \frac{\overline{\left(d \zeta_{t}\right)^{2}}}{\left|\left(d \zeta_{t}\right)^{2}\right|},\left(d \zeta_{t}\right)^{2}\right] } & =\int_{R_{t}} \frac{1}{2 t} \frac{\overline{\left(d \zeta_{t}\right)^{2}}}{\left|\left(d \zeta_{t}\right)^{2}\right|}\left(d \zeta_{t}\right)^{2} \\
& =\frac{1}{t} \sum_{j} \int \frac{i}{2} d \zeta_{j, t} \overline{d \zeta_{j, t}}=2 \pi \sum_{j}\left|c_{j}\right|^{1 / 2} \log r_{j}
\end{aligned}
$$

It follows from (2) and (3) that the length of the tangent vector $(1 / 2 t) \overline{\left(d \zeta_{t}\right)^{2} / \mid}\left(d \zeta_{t}\right)^{2} \mid$ is bounded above by 


$$
\frac{C_{1}}{t^{3 / 2}} \text { for } t \geqq 1, \quad C_{1}>0
$$

Since $\int_{1}^{\infty} d t / t^{3 / 2}$ converges, we see that this curve has finite length in the Weil-Petersson metric.

The Teichmüller distance between $R$ and $R_{t}$ is $\log t$ and since this metric is complete we see that for any sequence of $t$ 's tending to infinity the surfaces $R_{t}$ cannot converge. The Weil-Petersson and Teichmüller metrics induce the same topology and thus such a sequence cannot converge in the Weil-Petersson metric even though it is Cauchy. We conclude that the Weil-Petersson metric is not complete.

3. Further remarks. If $\dot{R}$ is obtained from $R$ by the removal of finitely many points and the upper half plane $U$ is taken as a finitely ramified cover of $\dot{R}$ then we again choose $\varphi d z^{2}$ as a JenkinsStrebel differential where in this case the possible poles of $\varphi d z^{2}$, the punctures of $\dot{R}$ as well as those points above which the cover is ramified are all considered as critical points. With this convention the branch points lie on the boundaries of the resulting annuli and the Poincare metrics of the annuli again provide a bound for the restriction to the annuli of the Poincare metric of the surface. In this way the proof is extended to the general case of the Teichmüller space of a finitely generated Fuchsian group of the first kind.

The curve under consideration is a geodesic in the Teichmuiller metric and is readily seen to be a "pinching" of a given Riemann surface as in the work of L. Bers, [5]. An immediate consequence of the present investigation is that the Weil-Petersson metric is not uniformly equivalent to the Teichmüller-Kobayashi or Carathéodory metrics. We also observe that the Teichmüller space of a 4 times punctured sphere is conformally equivalent to the unit disc. In this case the Weil-Petersson metric is not uniformly equivalent to the Bergman metric since the latter is the Poincaré metric which is complete.

\section{REFERENCES}

1. L. V. Ahlfors, The complex analytic structure of the space of closed Riemann surfaces, Analytic Functions by R. Nevanlinna et al., Princeton University Press, Princeton, N. J. (1960), 45-66.

2. - Some remarks on Teichmüller's space of Riemann surfaces, Ann. of Math., 74 (1961), 171-91.

3. - Curvature properties of Teichmüller space, J. Analyse Math., 9 (1961), 161-76.

4. L. Bers, Quasiconformal mappings and Teichmüller's theorem, Analytic Functions by R. Nevanlinna et al., Princeton University Press, Princeton, N. J., 1960, 89-119. 
5. - On boundaries of Teichmüller spaces and on Kleinian groups, I, Ann. of Math., 91 (1970), 570-600.

6. A. Douady, and J. Hubbard, On the density of Strebel differentials, preprint.

7. J. A. Jenkins, On the existence of certain general extremal metrics, Ann. of Math., 66 (1957), 440-53.

8. H. L. Royden, Automorphisms and isometries of Teichmüller space, Ann. of Math, Studies, 66 (1971), 369-83.

9. — Metrics on Teichmüller space, Springer Lecture Notes in Math., 400 (1974), 71-78.

10. - Invariant metrics on Teichmüller space, Contributions to Analysis by L. V. Ahlfors et al., Academic Press, New York, N. Y., 1974, 393-99.

11. K. Strebel, Quadratic Differentials, Stanford University lecture notes, 1973.

Received September 2, 1975 and in revised form October 30, 1975.

STANFORD UNIVERSITY 



\section{PACIFIC JOURNAL OF MATHEMATICS}

\section{EDITORS}

RICHARD ARENS (Managing Editor)

University of California

Los Angeles, California 90024

\author{
R. A. Beaumont \\ University of Washington \\ Seattle, Washington 98105
}

\section{J. DugundjI}

Department of Mathematics University of Southern California Los Angeles, California 90007

D. Gilbarg and J. Milgram

Stanford University

Stanford, California 94305

\section{ASSOCIATE EDITORS}
E. F. BECKENBACH
B. H. NeumanN
F. WOLF
K. YoshidA

\section{SUPPORTING INSTITUTIONS}

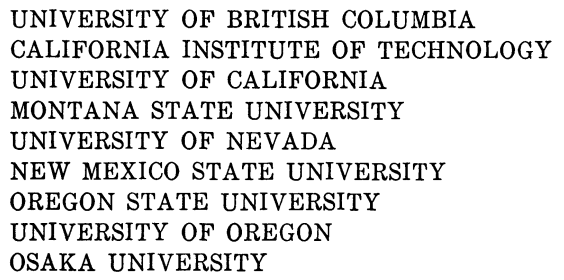

UNIVERSITY OF BRITISH COLUMBIA CALIFORNIA INSTITUTE OF TECHNOLOGY UNIVERSITY OF CALIFORNIA MONTANA STATE UNIVERSITY UNIVERSITY OF NEVADA NEW MEXICO STATE UNIVERSITY OSAKA UNIVERSITY OREGON STATE UNIVERSITY UNIVERSITY OF OREGON

\author{
UNIVERSITY OF SOUTHERN CALIFORNIA \\ STANFORD UNIVERSITY \\ UNIVERSITY OF TOKYO \\ UNIVERSITY OF UTAH \\ WASHINGTON STATE UNIVERSITY \\ UNIVERSITY OF WASHINGTON \\ AMERICAN MATHEMATICAL SOCIETY
}

The Supporting Institutions listed above contribute to the cost of publication of this Journal, but they are not owners or publishers and have no responsibility for its content or policies.

Mathematical papers intended for publication in the Pacific Journal of Mathematics should be in typed form or offset-reproduced, (not dittoed), double spaced with large margins. Please do not use built up fractions in the text of your manuscript. You may however, use them in the displayed equations. Underline Greek letters in red, German in green, and script in blue. The first paragraph or two must be capable of being used separately as a synopsis of the entire paper. Items of the bibliography should not be cited there unless absolutely necessary, in which case they must be identified by author and Journal, rather than by item number. Manuscripts, in triplicate, may be sent to any one of the editors. Please classify according to the scheme of Math. Reviews, Index to Vol. 39. All other communications should be addressed to the managing editor, or Elaine Barth, University of California, Los Angeles, California, 90024.

The Pacific Journal of Mathematics expects the author's institution to pay page charges, and reserves the right to delay publication for nonpayment of charges in case of financial emergency.

100 reprints are provided free for each article, only if page charges have been substantially paid. Additional copies may be obtained at cost in multiples of 50 .

The Pacific Journal of Mathematics is issued monthly as of January 1966. Regular subscription rate: $\$ 72.00$ a year $(6$ Vols., 12 issues). Special rate: $\$ 36.00$ a year to individual members of supporting institutions.

Subscriptions, orders for back numbers, and changes of address should be sent to Pacific Journal of Mathematics, 103 Highland Boulevard, Berkeley, California, 94708.

PUBLISHED BY PACIFIC JOURNAL OF MATHEMATICS, A NON-PROFIT CORPORATION

Printed at Kokusai Bunken Insatsusha (International Academic Printing Co., Ltd.), 8-8, 3-chome, Takadanobaba, Shinjuku-ku, Tokyo 160, Japan. 


\section{Pacific Journal of Mathematics}

\section{Vol. 61, No. 2 December, 1975}

Graham Donald Allen, Francis Joseph Narcowich and James Patrick Williams, An operator version of a theorem of Kolmogorov .......................

Joel Hilary Anderson and Ciprian Foias, Properties which normal operators share with normal derivations and related operators . . . . . . . . . . . . . . . . . . . . .

Constantin Gelu Apostol and Norberto Salinas, Nilpotent approximations and

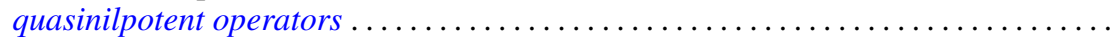

James M. Briggs, Jr., Finitely generated ideals in regular $F$-algebras . . . . . . . . . . .

Frank Benjamin Cannonito and Ronald Wallace Gatterdam, The word problem and power problem in 1-relator groups are primitive recursive ..................

Clifton Earle Corzatt, Permutation polynomials over the rational numbers ...........

L. S. Dube, An inversion of the $S_{2}$ transform for generalized functions . . . . . . . . . . William Richard Emerson, Averaging strongly subadditive set functions in unimodular

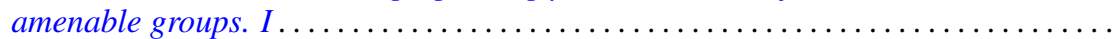

Barry J. Gardner, Semi-simple radical classes of algebras and attainability of

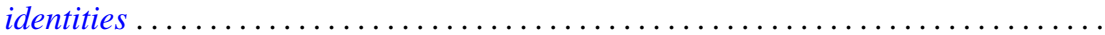

Irving Leonard Glicksberg, Removable discontinuities of A-holomorphic functions ....

Fred Halpern, Transfer theorems for topological structures . . . . . . . . . . . . . . .

H. B. Hamilton, T. E. Nordahl and Takayuki Tamura, Commutative cancellative

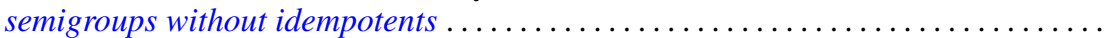

Melvin Hochster, An obstruction to lifting cyclic modules .....................

Alistair H. Lachlan, Theories with a finite number of models in an uncountable power

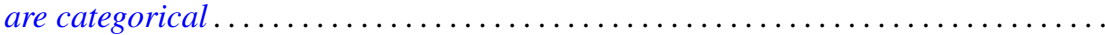

Kjeld Laursen, Continuity of linear maps from $C^{*}$-algebras . . . . . . . . . . . . .

Tsai Sheng Liu, Oscillation of even order differential equations with deviating arguments ....

Jorge Martinez, Doubling chains, singular elements and hyper- $Z$

Mehdi Radjabalipour and Heydar Radjavi, On the geometry of num Thomas I. Seidman, The solution of singular equations, I. Linear equations in Hilbert

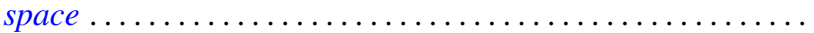

R. James Tomkins, Properties of martingale-like sequences ......

Alfons Van Daele, A Radon Nikodým theorem for weights on von Neumann

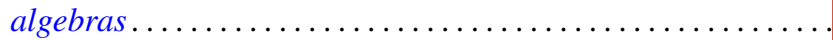

Kenneth S. Williams, On Euler's criterion for quintic nonresidues

Scott Andrew Wolpert, Noncompleteness of the Weil-Petersson metric for Teichmüller

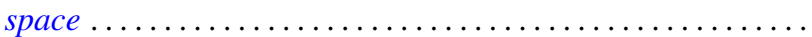

Volker Wrobel, Some generalizations of Schauder's theorem in locally convex

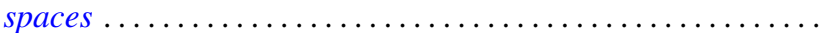

Kelly Denis McKennon, Corrections to: "Multipliers of type $(p, p)$ "; "Multipliers of type $(p, p)$ and multipliers of the group $L_{p}$-algebras"; "Multipliers and the

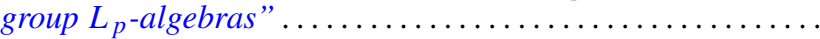

Andrew M. W. Glass, W. Charles (Wilbur) Holland Jr. and Stephen H. McCleary, Correction to: " $a *$-closures to completely distributive lattice-ordered

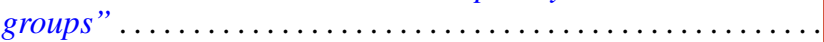

Zvi Arad and George Isaac Glauberman, Correction to: "A characteristic subgroup of

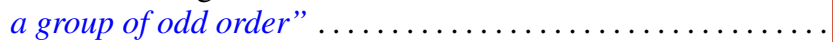

Roger W. Barnard and John Lawson Lewis, Correction to: "Subordination theorems

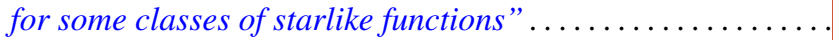

